\title{
Gaucher Disease: Clinical, Biological and Therapeutic Aspects
}

\author{
Azza Dandana Souhaira Ben Khelifa Hinda Chahed Abdelhédi Miled \\ Salima Ferchichi \\ Laboratory of Biochemistry, Farhat Hached Hospital, Sousse, Tunisia
}

\section{Key Words}

$\beta$-Glucocerebrosidase - Lysosomal storage disease .

Gaucher disease - Therapeutic aspect

\begin{abstract}
We present a brief review of Gaucher disease (GD), the most common lysosomal storage disease. GD is a rare autosomal recessive disorder characterized by the defective function of the catabolic enzyme $\beta$-glucocerebrosidase (GBA), leading to an accumulation of its substrate, glucocerebroside. Clinical signs and symptoms include neurological dysfunctions, bone infarcts and malformations, hepatosplenomegaly and hypersplenism leading to anemia, neutropenia and thrombocytopenia. Enzyme replacement therapy with recombinant GBA is the mainstay of treatment for GD, which became the first successfully managed lipid storage disease. Future treatments may include oral enzyme replacement and/or gene therapy interventions.

๑) 2015 S. Karger AG, Basel
\end{abstract}

\section{Introduction}

Lysosomal storage disease (LSD) describes a heterogeneous group of rare inherited disorders characterized by the accumulation of undigested or partially digested mac- romolecules, which ultimately results in cellular dysfunction and clinical abnormalities. Organomegaly, connective tissue and ocular pathology may result, as well as central nervous system dysfunction.

Classically, LSDs encompassed only enzyme deficiencies of the lysosomal hydrolases. More recently, the concept of LSD has been expanded to include deficiencies or defects in proteins necessary for the normal posttranslational modification of lysosomal enzymes (which themselves are often glycoproteins), activator proteins, or proteins important for proper intracellular trafficking between the lysosome and other intracellular compartments.

\section{Classification of LSD}

The classification of the LSDs is based either on the deficient enzyme or on the chemical composition of the storage material. In terms of the storage material, LSDs can be divided into three large groups - the sphingolipidoses, mucopolysaccharidoses and glycoproteinoses and several other individual entities. Most LSDs are caused by deficiencies of enzymes that degrade carbohydrate side chains and their storage materials are carbohydrates or other glycocompounds. Table 1 gives a simplified classification of the most common LSDs.

\section{KARGER}

E-Mail karger@karger.com www.karger.com/pat
C 2015 S. Karger AG, Basel

1015-2008/15/0831-0013\$39.50/0
Dr. Azza Dandana

Laboratory of Biochemistry, Farhat Hached Hospital

Doctor Moreau Street

Sousse 4000 (Tunisia)

E-Mail azzadandana@yahoo.fr 
Table 1. Classification of LSD

\begin{tabular}{lll}
\hline LSD & Deficient enzyme & Phenotype \\
\hline $\begin{array}{l}\text { Sphingolipidoses } \\
\text { GM1 gangliosidosis }\end{array}$ & & neuronal lipidosis mucopolysaccharidosis \\
GM2 gangliosidosis (Tay-Sachs disease) & h-galactosidase & hexosaminidase A \\
Niemann-Pick disease & sphingomyelinase & neuronal lipidosis \\
Globoid cell leukodystrophy (Krabbe disease) & galactocerebrosidase & leukodystrophy \\
Metachromatic leukodystrophy & arylsulfatase A & leukodystrophy \\
GD & glucocerebrosidase & storage histiocytosis \\
Mucopolysaccharidoses & glycosaminoglycan cleaving enzymes & mucopolysaccharidosis \\
Glycoproteinoses & glycoprotein cleaving enzymes & mucopolysaccharidosis \\
Glycogenosis type II (Pompe disease) & a-glucosidase & skeletal and cardiac myopathy \\
Neuronal ceroid lipofuscinosis & lysosomal proteases & neuronal lipidosis \\
\hline
\end{tabular}

\section{Clinical Presentations and Diagnosis}

Several clinical manifestations, such as hepatosplenomegaly, coarse facial features and skeletal dysplasia, can serve as an important clue which should lead to the consideration that the patient's problems are likely to be due to LSD. Diagnostic confirmation necessitates biochemical and/or molecular genetic testing. Characterization of the gene defects in an affected individual has revealed the occurrence of several distinct mutations. Although this phenomenon partly explains differences in disease severity, extensive studies to examine the relationship between genotype (gene defect) and phenotype (clinical expression) have often shown the lack of perfect concordance. Unfortunately, this is not the case with LSDs, in which the diagnosis when suspected often necessitates testing for the presence of excess substrates in body fluids or the activity of several different enzymes in blood or tissues [1].

\section{Outline of the History of Gaucher Disease}

Gaucher disease (GD) is caused by a functional deficiency of the acid hydrolase, $\beta$-glucocerebrosidase (GBA), or glucosylceramide (EC 3.2.1.45) [2,3]. The disease was first described by Phillipe Gaucher in his doctoral dissertation in 1882. Twenty years later, Nathan Brill proved its autosomal recessive inheritance and used its eponymic name. In the 1920s, the neuronopathic phenotype of the disease was described. In the 1960s, Roscoe Brady established that the pathomechanism of GD stems from the deficiency of GBA activity [4].

\section{Pathomechanism}

The insufficient catabolism of glucosylceramide and the engorgement of macrophages by this substrate lead to visceral manifestations of GD. Sphingolipids have been implicated in inflammatory and apoptotic processes [5] and glucosylceramide might have direct activating or enhancing effects on macrophage function [6], possibly mediated through selective calcium channel dysregulation. Indeed, several indicators of macrophage activation, including chitotriosidase, CCL18, angiotensin-converting enzyme [7] and cathepsin S, have been identified in excess in the plasma of patients with GD. Histological assessment showed that such proinflammatory molecules, including tumor necrosis factor- $\alpha$, are variably increased in some splenic Gaucher cells [8]. An alternative mechanism by which these proinflammatory and anti-inflammatory pathways could be activated is through abnormal folding of mutant proteins in the endoplasmic reticulum [9]. Such abnormal folding initiates an unfolded protein response that can trigger apoptotic or inflammatory pathways in various tissues [10]. Evidence suggests that some mutations in GD might lead to proteins that are abnormally folded or are not properly trafficked, or both. Although direct evidence of unfolded protein response involvement is not available for GD, this pathway seems to be active in GM1 gangliosidosis [11].

Thus, the development of alternative agents to alter the unfolded protein response could be on the horizon for patients with GD in whom this mechanism is activated. By comparison with visceral tissues, the pathogenesis of the brain disease is completely different. A toxic reaction to a low-level substrate, glucosylsphingosine, might be the inciting mechanism [12]. This mechanism has come under question, since glucosylceramide seems more im- 
Table 2. Clinical classification of GD

\begin{tabular}{|c|c|c|c|}
\hline \multirow{2}{*}{ Affected groups } & Most common in Ashkenazi Jewish & No particular ethnicity & No particular ethnicity \\
\hline & population ( 1 in 450$)$ & 1 in 100,000 live births & 1 in 50,000 live births \\
\hline Distinguishing & Liver, spleen, and bone & Early nervous system problems & Later onset of nervous system \\
\hline symptom & No nervous system problems & Brainstem abnormalities & $\begin{array}{l}\text { problems: incoordination, mental } \\
\text { deterioration, myoclonic seizures }\end{array}$ \\
\hline Effects of disease & Varies from mild to severe & Death in infancy (age $<2$ years) & $\begin{array}{l}\text { Slowly progressive - becomes severe } \\
\text { later in childhood }\end{array}$ \\
\hline $\begin{array}{l}\text { Glucocerebrosidase } \\
\text { activity }\end{array}$ & $\begin{array}{l}\text { Some activity, but much less than } \\
\text { normal }\end{array}$ & Very little activity & Little activity \\
\hline
\end{tabular}

portant than glucosylsphingosine for the induction of abnormal calcium fluxes in the endoplasmic reticulum with potential disruptive effects in cells [13]. Disruption of the ceramide-to-glucosylceramide ratio can affect barrier formation in the epidermal layer of the skin, leading to ichthyosis or a collodion skin presentation in individuals who are severely affected (those with type 2) [14].

\section{Types of $G D$}

The symptoms associated with GD are due to the progressive accumulation of Gaucher cells in various organs. Thus, GD is a multisystemic disorder with disease manifestation at all ages dependent on the subtype of GD. Three basic clinical forms of GD can be distinguished depending on the degree of neurological involvement; however, today the different forms of GD are considered to reflect a continuum ranging from early-onset to late-onset disease, and from severe forms with neurological symptoms to mild forms with solely visceral manifestations [15]. Type $1 \mathrm{GD}$ is the most frequent form and accounts for $94 \%$ of all registered GD cases according to the Gaucher Registry [16]. It leads to a chronic course of disease and the organs frequently affected are the spleen, liver, bone marrow and, in severe cases, also the lung and kidney. Hepatosplenomegaly and hematological complications, including anemia and thrombocytopenia with bleeding, are common in untreated type $1 \mathrm{GD}[17,18]$. Acute neuronopathic GD (type 2 GD) manifests in early childhood, neurological deterioration progresses quickly and death generally occurs before the age of 2 years. Subacute neuronopathic GD (type 3) shows a slower neurological involvement and usually occurs in adolescence [15] (table 2).

Clinical Aspects of GD

\section{Diagnosis of GD}

GD may also come to light as a result of investigations for pancytopenia or visceromegaly; thus, Gaucher cells may be identified on tissue biopsy specimens, principally of bone marrow (during investigations for splenomegaly or cytopenias) or liver (during the course of investigations for hepatomegaly or abnormal liver-related biochemical tests). Specific diagnosis is, however, made by measuring acid $\beta$-glucosidase activity in fresh peripheral blood leukocytes or occasionally by enzymatic analysis of fibroblasts cultured from skin biopsy specimens. Confirmation and better characterization of the condition may subsequently be afforded by molecular analysis of the human GBA gene, which encodes lysosomal GBA [19].

Residual activity of the enzyme depends on the type of mutation change in the gene for GBA. N370S and L444P are the most frequent mutations in the gene for GBA located on chromosome 1q21. The mutation N370S occurs only in the Caucasian population, which, in time, may prove the existence of founder effect. The presence of this mutation even on a single allele ensures the residual activity of the enzyme sufficient for correct catabolism of glucocerebroside in neurons and, hence, the lack of neurological symptoms. The mutation L444P is panethnic; homozygosity for this mutation leads to the neuronopathic form of the disease and occurs in more than $70 \%$ of patients. The frequency of the neuronopathic form is estimated to be 1 per 40,000 live births. It occurs much more frequently in the Ashkenazi population, with an incidence being 1 per 1,000 live births, where N370S (57-70\%) and $84 \mathrm{GG}(10 \%)$ are the most frequent mutations [20]. 
Fig. 1. GBA structure and mutation distribution. a The $62-\mathrm{kb}$ region surrounding the GBA along chromosome 1q showing the known genes and pseudogenes and their transcription direction. C1orf2 = Chromosome 1 open reading frame 2 (cote1); MTXP = metaxin 1 pseudogene; GBAP = glucocerebrosidase pseudogene; MTX1 = metaxin 1; THBS3 = thrombospondin 3. b The exonic structure of GBA, with the two ATGs and positions of 15 common mutations indicated. $c$ The number of reported substitution, deletion, insertion and splice-site mutations per exon [23].

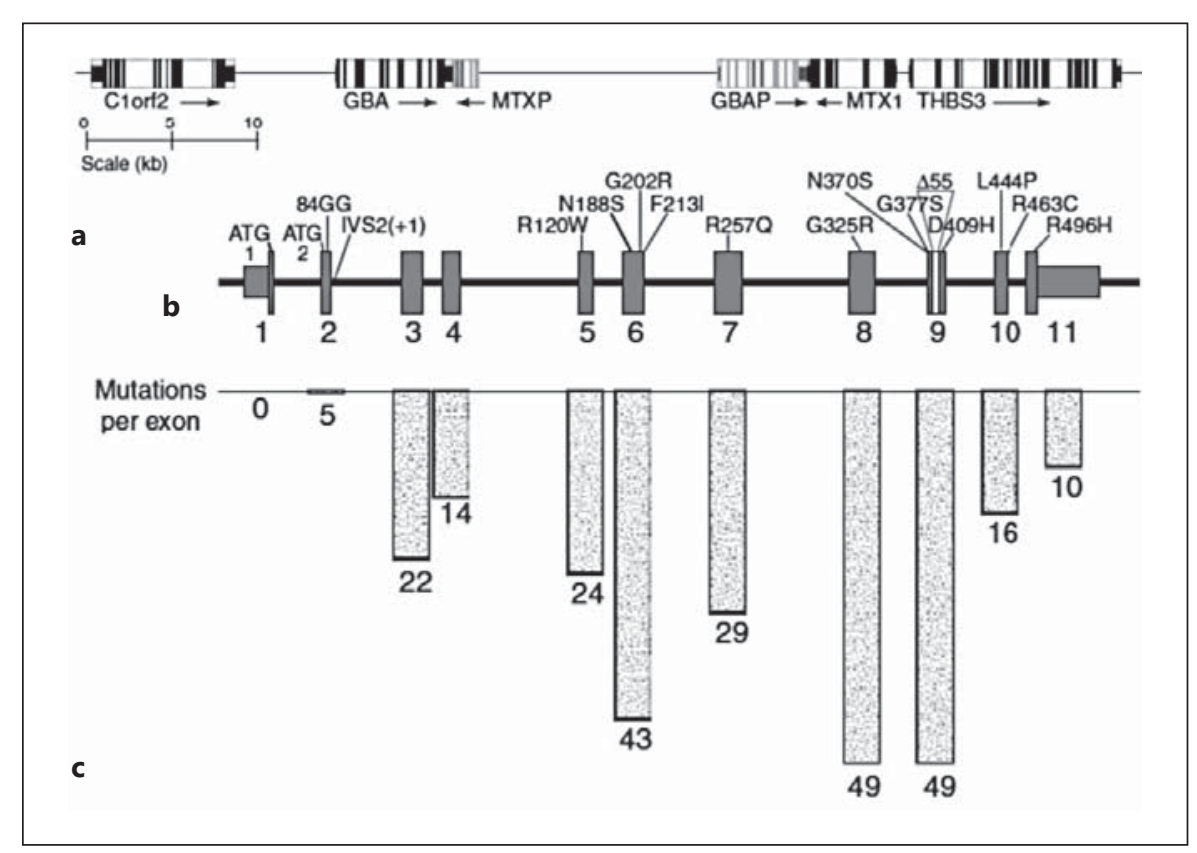

\section{Mutations and Polymorphism-Defined Mutations}

Presently, nearly 300 mutations have been identified in Gaucher patients, including frame-shift mutations, point mutations, deletions, insertions, splice site mutations and recombinant alleles (fig. 1). Recombination events with a highly homologous pseudogene downstream of the GBA locus have also been identified, resulting from gene conversion, fusion or duplication (fig. 2). The identification of mutant GBA alleles can be problematic, as primers must be designed to discriminate between the functional gene and the pseudogene. Initially, laboratories used different PCR-based screening techniques to identify a limited panel of two to seven known mutations. Among type 1 patients of Ashkenazi Jewish ancestry, this was a fairly efficient approach, detecting approximately $90 \%$ of mutant alleles with a screen for five to six mutations [21-23]. In non-Jewish populations, however, this screening strategy did not identify a significant portion of mutations, especially in patients with neuronopathic GD. The wider incorporation of automated sequencing to genotype Gaucher patients has led to the identification of many new GBA mutations [24] (fig. 1).

\section{Recombinant and Complex Alleles}

A prevalent class of mutant alleles encountered in patients with GD is due to recombination events [25-29]. Such alleles have been designated as 'complex' [27], 'pseudopattern' or 'psi' [26], 'rec' for 'recombinant' [25], 'fu- sion' [30] and 'chimeric' alleles [31]. Recombination within the glucocerebrosidase locus appears to be enhanced by the high degree of sequence identity and the close physical proximity of the GBA pseudogene. In fact, the sites of these recombination events are variable [32, 33, 29], ranging from intron 2 to exon 11 (fig. 2). In addition, the mechanisms of recombination differ; it has been shown that recombinant GBA alleles arise from both reciprocal and nonreciprocal recombination [29]. Figure 2 illustrates the approximate sites of recombination, extent of incorporated pseudogene sequence and associated amino acid changes for different groups of these alleles. Other complex alleles that do not appear to be derived from the pseudogene have also been identified. These may arise as disease-causing alterations in cis with polymorphisms, or may represent multiple pathogenic substitution events. While initially reported as single disease-causing mutations [34, 25], two specific alterations, c.1093G4C (E326 $\mathrm{K})$ and c.1223C4T (T369 M), have been found in patients primarily in cis with other identified mutations $[35,36]$ and have led to published corrections after identification of a second mutation on the same allele [37, 38] (fig. 2).

\section{Polymorphisms}

Several different GBA polymorphisms have been described. Early characterization of the GBA demonstrated a restriction fragment length polymorphism by digestion with PvuII, alleles that generated a 1.1-kb fragment were
16

Pathobiology 2016;83:13-23 DOI: $10.1159 / 000440865$
Dandana/Ben Khelifa/Chahed/Miled/ Ferchichi 


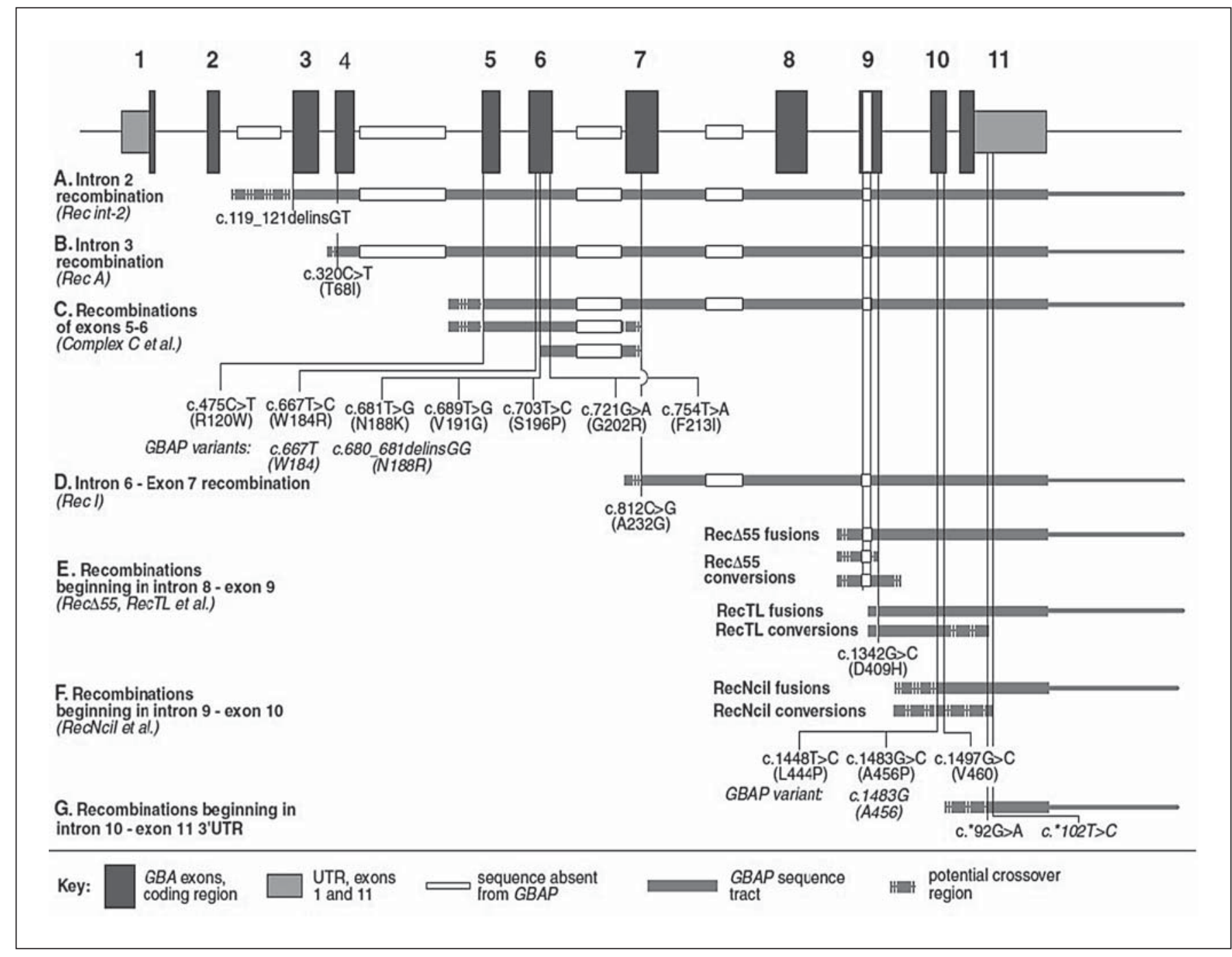

Fig. 2. Recombinant alleles observed in GBA. The exonic and intronic structure of GBA is shown. Typical nonreciprocal (gene conversion) and reciprocal (gene fusion or duplication) recombinants are shown. The exact locations of the break points have not been mapped for many recombinant alleles. Regions of sequence identity between GBA and GBAP that flank the observed mis- matches in which recombination could occur are depicted as barred lines. Pseudogene sequence variations that can alter the combination of amino acid changes seen in a recombinant allele are shown in italics [39]. The figure was reprinted with permission from Sorge et al. [40]. designated Pv1.11 and those which lacked this fragment were designated Pv1.1 [39]. In time, this polymorphism was identified as a g.4813G4A substitution in intron 6 [40] and expanded to include 11 additional polymorphisms, 8 in intronic sequences and 3 in the 50-untranslated region. Each of these sequence variations are in linkage disequilibrium and constitute two major haplotypes [41]. Mutation c.1226A4G (N370S) is in linkage disequilibrium with the Pv1.1 haplotype [40, 42-46], while c.84dupG is found with Pv1.11 [40, 41, 46]. Another GBA polymorphism, a g.5470G4A transition of the Pv1.1 haplotype, appears to be limited to the Portuguese and Spanish populations $[42,47]$. In addition, a polymorphic CT repeat (5GC3.2) $3.2 \mathrm{~kb}$ upstream and a tetranucleotide AAAT repeat (ITG 6.2) $9.8 \mathrm{~kb}$ downstream of GBA have been identified [48]. Variation in the GBA pseudogene includes 17 single-nucleotide substitutions, a three-nucleotide deletion and a polyadenine tract $[49,47]$. These polymorphisms in GBAP are important in the recognition of GBAP to GBA gene conversion events [47]. 


\section{GD and Parkinson's Disease}

Several lines of evidence suggest an association between Parkinsonism and mutations in the gene encoding the lysosomal enzyme glucocerebrosidase (GBA), which is deficient in patients with GD [50]. Parkinson's disease (PD) is the second most common neurodegenerative disorder, with greater than $1 \%$ affected over 65 years of age and more than $4 \%$ of the population affected by the age of 85 years [51, 52].

A histopathological study of the brains of patients with GD has shown Lewy bodies in the hippocampal regions and in several other scattered regions throughout the brain [53]. It is unclear whether the Lewy bodies occur because of localized accumulations of glucosylceramide or an abnormal folding of specific mutant proteins in specific regions of the brain. This association of GD alleles (homozygotes or heterozygotes) and parkinsonism is weak and quite controversial [54], but the apparent overrepresentation of these alleles in the brains of patients with PD suggests a stronger association than might have been appreciated from epidemiological data [55].

Recognition of the association between GBA mutations and parkinsonism began in the clinic, with the identification of rare patients with GD who also had parkinsonian symptoms [56]. Moreover, pedigree analyses indicated an elevated incidence of PD in the relatives of Gaucher patients, many of whom were obligate heterozygotes $[56,57]$.

In 2009, Sidransky et al. [50] published a hallmark study on this topic: a collective analysis of 5,691 patients with PD complemented by 4,898 controls from 16 centers across 12 countries. For the pool of participants in which the full GBA coding region was screened, loss-offunction mutations were observed in $6.9 \%$ of cases and in $1.3 \%$ of the controls (odds ratio 5.4; 95\% CI 3.9-7.6). Among the Ashkenazi Jewish subset, higher mutation frequencies were seen: $19.3 \%$ in cases and $4.1 \%$ in controls $[56,50]$.

The findings were not exclusive to a specific ethnicity, nor associated with any particular GBA mutation. Additional noted trends were that subjects carrying mutations presented an average of 4 years earlier, were more likely to have a family history of $\mathrm{PD}$, and had less bradykinesia and rest tremor and more cognitive changes described [50]. Other cohort studies have corroborated the results from this collaborative examination, reinforcing mutations in $G B A$ as the number one genetic risk factor for $\mathrm{PD}$ $[50,57,58]$.

\section{Therapeutics of GD}

Skeletal complications are a major cause of morbidity in patients with GD type $1[59,60]$. On magnetic resonance imaging, areas of reduced bone marrow signal intensity ('dark marrow') occur in T1-weighted sequences. Progressive accumulation of Gaucher cells displaces normal adipocytes from the marrow compartment $[60,61]$, leading to abnormal quantities and the distribution of 'dark marrow'. This process begins in the axial skeleton and progresses to the appendicular skeleton, advancing in the lower extremity in a predictable sequence over time [60]. Focal collections of Gaucher cells may result in lytic lesions. The mechanistic link between marrow infiltration and the development of bony complications is not clear, but extensive Gaucher cell infiltration is associated with osteopenia, bone infarctions, avascular necrosis, lytic lesions and pathological fractures [61, 62]. Chronic bone pain and acute bone crises also often accompany skeletal disease in GD type 1 patients.

Enzyme replacement therapy (ERT) and substrate reduction therapy have been demonstrated to have beneficial effects on bone pain, bone crises and the extent of osteoporosis [62]. In the 2 decades since the introduction of ERT, it has become abundantly clear that many of the symptoms and signs of visceral GD, such as hepatosplenomegaly, as well as anemia, thrombocytopenia and often skeletal or lung involvement, will respond adequately to ERT. While ERT forms the mainstay of treatment for type I GD, the iminosugar miglustat (Zavesca ${ }^{\circledR}$; Actelion Pharmaceuticals Ltd.) represents an alternative treatment strategy - substrate reduction therapy [63]. In clinical trials in ERT-naïve adults with type I GD, miglustat treatment was effective in reducing liver and spleen volume and increasing hemoglobin concentration and platelet count during 12-36 months of treatment [64-67]. Miglustat has also been shown to improve bone mineral density in both the trabecular and cortical bones [68]. Although the controversy of what is the minimally effective dose and/or optimal dosage schedule has not been resolved, it appears that, regardless of dosage, the responsiveness of all major disease features and surrogate markers will plateau after 3-5 years and most patients remain 'stable' while maintaining the same ERT schedule $[68,69]$. In June 2009 , the Genzyme Corporation announced a viral contamination at its manufacturing site that involved the production of imiglucerase. In November 2009, Genzyme advised physicians of foreign particle contamination associated with certain batches of imiglucerase. The dramatic reduction in global enzyme availability led to early access
18

Pathobiology 2016;83:13-23 DOI: $10.1159 / 000440865$
Dandana/Ben Khelifa/Chahed/Miled/ Ferchichi 
or compassionate use programs at the request of the regulatory agencies for two new ERTs for GD that were at the time in phase III trials: velaglucerase alfa (VPRIVTM; Shire HGT, Cambridge, Mass., USA) and taliglucerase alfa (Elelyso ${ }^{\mathrm{TM}}$; Protalix Biotherapeutics, Karmiel, Israel) [69]. The early access program of velaglucerase alfa was initiated in Israel in November 2009. At that time, the shortage of imiglucerase was not acute locally, but because of the concern about foreign particle contamination, previously treated patients, some with newly emerging symptoms such as fatigue and bone pain, were interested in restarting ERT; similarly, there were some naïve-to-treatment patients who opted to receive velaglucerase alfa rather than await the resolution of the imiglucerase supply issue [69].

On the other hand, eliglustat is an example of an investigational oral substrate reduction therapy for adults with GD type 1. Eliglustat is pharmacologically distinct from ERT, the current standard of care for GD type 1 [70]. ERT supplies exogenous acid $\beta$-glucosidase to break down accumulated glucosylceramide. Eliglustat, a ceramide analog, inhibits glucosylceramide synthase, thereby reducing the synthesis of its substrate, glucosylceramide, to balance production with the impaired rate of degradation. The efficacy, safety and tolerability of eliglustat after 1 and 2 years of treatment were demonstrated in a phase 2 trial of treatment-naïve adult patients with GD type 1 [71].

The long-term follow-up of eliglustat treatment for previously untreated GD type 1 patients demonstrated continuation and maintenance of improvements in hematological parameters, organ volumes, disease-related biomarkers and bone parameters. Phase 3 clinical trials in progress will further elucidate the efficacy and safety of eliglustat in a larger population of treatment-naïve GD type 1 patients as well as in GD type 1 patients previously stabilized on ERT [62].

Enzyme therapy also has disadvantages, including that manufacture is costly and intravenous infusions are inconvenient to administer. Drug infusions, usually given every 2-4 weeks, may be painful for some patients and could render patients unnecessarily dependent on hospital services. Also, young children may develop a fear of needles and an aversion to the treatment. In countries with underdeveloped health care systems, intravenous infusion may constitute an elaborate procedure, particularly where clean needles and appropriate equipment for drug administration under safe circumstances in public health services are not easily available. Ultimately, therapeutic compounds able to safely enter the brain and nervous system by traversing the blood-brain barrier will offer an immense benefit and will revolutionize the field [72].
Pharmacological chaperone (PC) therapy is a new strategy to increase residual activity by stabilizing misfolded mutant proteins, preventing endoplasmic-reticulum-associated degradation in proteasomes and allowing trafficking to lysosomes $[73,74]$. This approach is especially applicable in GD because only a modest increase in residual GBA should be sufficient to ameliorate the phenotype. Moreover, these small molecules should be able to cross the blood-brain barrier [75].

The first clinical trial of PC used isofagomine tartrate (Amicus Therapeutics) [72], but phase 2 trials failed to meet the end points, and further development was abrogated. An alternative PC is ambroxol hydrochloride (ExSAR Corporation) [76], originally developed as a mucolytic agent 30 years ago (Mucosolvan; Boehringer-Ingelheim) and available over the counter in many countries. Ambroxol has also been used in the treatment or prophylaxis, or both, of neonatal respiratory distress syndrome [77]. It is to be hoped that among these PC medications there will be at least one that will ameliorate neuronopathic features.

Although gene therapy [78] is theoretically promising, there is no ethical justification to experiment on patients who have alternative, safe and effective treatment. However, stem cell therapy with a low-risk conditioning regimen [79] may have a role in GD and other metabolic disorders but, again, there is the challenge of affecting neurological features [80].

The therapeutic approach that is just on the horizon is the use of induced pluripotent stem cell-derived stem cell transplantation. For GD and other lysosomal disorders, wild-type donor bone marrow transplantation has been used because monocytes from the peripheral blood can migrate across the blood-brain barrier and become CNS microglial cells that could affect metabolic cross-correction [80]. For GD, bone marrow or stem cell transplantation has not been effective for the central nervous system disease because of the lack of a secretable enzyme. Induced pluripotent stem cells are an attractive alternative for generating either hematological progenitor cells or neural progenitor cells for direct cellular and/or enhanced gene therapy $[80,81]$.

\section{Epidemiology of GD in General and in Tunisian Populations}

The birth frequency of GD is approximately 1:60,000 live births in the general population. However, genetic studies indicate a homozygote frequency of approximate- 
ly 1:950 Ashkenazi Jews, many of whom appear to remain asymptomatic [75]. GD is rare in the Tunisian population, with an estimated frequency of 1 per 108,000 [19]. In Tunisia, where $98 \%$ of the population is Arab, a series of 27 patients was reported over an 18-year period: 20 patients with type I disease, 3 patients each with type II and type III disease, and 1 patient whose genotype was unknown [82]. Further studies have concluded that GD is not exceptional in Tunisia, reflecting the finding that the natural course of GD was clinically comparable to that described in other non-Arab patients [83].

In order to identify the mutation spectrum causing GD in Tunisian patients, 30 patients ( 12 females and 18 males) from 25 unrelated families originating from various geographic locations in Tunisia were investigated. Fourteen among 25 families (56\%) were consanguineous. Based on the absence of neurological involvement, all of the patients were clinically classified as GD type 1, except for 2 patients who were diagnosed as types 2 and 3 [84].

The N370S is the most frequent mutation among Tunisian patients with GD, as is the case in European populations, with frequencies similar to Southern European countries, i.e. $55 \%$ for Spanish patients [85] and 36.5\% for Italian patients [86]. The age estimation of N370S suggests that this mutation occurred first in non-Jewish and then passed into the Jewish population [87]. Consequently, it is not surprising that the N370S mutation is also relatively frequent in the southern Mediterranean due to geographical and historical reasons. This mutation was likely introduced into North Africa during the Roman Empire. It would be very interesting to analyze the haplotype of Tunisian patients carrying the N370S mutation to determine the population migratory flows. The recombinant allele RecNciI was found to be the third most frequent mutation. Its frequency in Tunisian patients is higher than in Italian patients (2.1\%) [18], and this complex allele was observed in combination with N370S in 7 patients with a nonneuronopathic form of GD. This genotype (N370S/RecNciI) is the most frequent one in nonconsanguineous families (6/11 nonconsanguineous families). The rate of consanguinity among the investigated patients (56\%) is relatively low compared to other recessive diseases investigated so far in Tunisia, which could increase to up to $80 \%$ among affected families. For this reason, we think that the N370S and RecNciI alleles should be relatively frequent in the general population [87].

On the other hand, Ben Turkia et al. [88] reported the first Tunisian experience with ERT of pediatric nonneuronopathic GD. Two children presented as severe viscer- al or hematological cases - one had myocardiopathy and primitive portal hypertension, and the other was diagnosed with cirrhosis related to GD. Recurrent avascular necrosis and osteoporosis have also justified treatment in another child. All 3 patients received an initial dose of 60 $\mathrm{U} / \mathrm{kg} / 2$ weeks. A gradual disappearance of hepatosplenomegaly and a rapid normalization of hematological parameters were observed in 2 of the patients, whilst a resistance to treatment indicated splenectomy in the other patient. The improvement in bone mineral density was slower. A significant growth gain was observed in the patients with growth retardation and no patient developed Cerezyme antibodies. Despite its effectiveness and safety being demonstrated in these children, ERT remains inaccessible because of its cost in emerging countries. Allogeneic bone marrow is an alternative therapy to encourage and to propose precociously for severe pediatric forms of GD [88].

The study by Ben Rhouma et al. [89] described the first report of an association between GD and PD in Tunisian adult patients with GD. In their study, 2 patients were homozygous for the N370S mutation and 1 patient developed PD at the age of 52 years. So far, this mutation has been found in $44 \%$ of pediatric Tunisian patients with GD [84]. Unlike most of the autosomal recessive genetic diseases in Tunisia, of which the majority of patients are born to consanguineous marriages and which are homozygous for deleterious alleles, the N370S mutation is encountered in a heterozygous state in combination with other mutated alleles [89]. The first report describing adult patients with GD in Tunisia was published by our laboratory [90] and included a 50-year-old patient with GD without any neurological disorder. Many adult cases with GD have been reported worldwide. Based on data from the Gaucher Registry (ICGG 2008), the N370S mutation is found in $53 \%$ of all GD patients, which represents the highest prevalence. About $14 \%$ of GD patients are diagnosed between the age of 31 and 50 years.

In Tunisia, like other autosomal recessive disorders, GD is rare and does not represent a major public health concern. There is a great variability in the clinical manifestation as well as in the onset and course of GD. This supports the need for a new classification and reinforces the hypothesis of the concept of a phenotypic continuum. Clinicians, especially in hematology and internal medicine, should suspect the diagnosis of GD in any adult patient suffering from abdominal and/or bone pain with an unexplained hematological disorder, especially in combination with organomegaly.
Dandana/Ben Khelifa/Chahed/Miled/ Ferchichi 


\section{Conclusion}

GD phenotypes associated with Parkinsonian syndrome, pulmonary hypertension and cancer are uncommon events in the patient population as a whole. Therefore, the mechanism behind the relationship between GBA mutations and PD or dementia with Lewy bodies remains elusive. The identification of different genetic modifiers and the mechanisms of their effects on the phenotypes seen in GD will significantly improve our understanding of genotype-phenotype relationships both in GD and in other Mendelian disorders.

\section{Acknowledgement}

The authors thank the staff of the Biochemistry Laboratory for their valuable contribution in this work.

\section{Disclosure Statement}

The authors have no financial, political, personal, religious, ideological, academic, intellectual, commercial or other competing interests to declare in relation to this paper.

\section{References}

1 Wraith JE: The clinical presentation of lysosomal storage disorders. Acta Neurol Taiwan 2004;13:101-106.

2 Brady RO, Kanfer JN, Shapiro D: Metabolism of glucocerebrosides. II. Evidence of an enzymatic deficiency in Gaucher's disease. Biochem Biophys Res Commun 1965;18:221225.

3 Beutler E, Grabowski GA: Gaucher disease; in Scriver CR, Beaudet AL, Sly WS, Valle D (eds): The Metabolic and Molecular Basis of Inherited Disease, ed 8. New York, McGrawHill, 2001, vol III, pp 3635-3668.

4 Beutler E, Grabowski G: Glucosylceramidelipidoses: Gaucher disease; in Scriver CR, Beaudet AL, Sly WS (eds): The Metabolic and Molecular Bases of Inherited Diseases. New York, McGrew-Hill, 1995, pp 2641-2670.

5 Bieberich E, Freischutz B, Suzuki M, et al: Differential effects of glycolipid biosynthesis inhibitors on ceramide-induced cell death in neuroblastoma cells. J Neurochem 1999;72: 1040-1049.

6 Gordon S, Taylor PR: Monocyte and macrophage heterogeneity. Nat Rev Immunol 2005; 5:953-964.

7 Brinkman J, Wijburg FA, Hollak CE, et al: Plasma chitotriosidase and CCL18: early biochemical surrogate markers in type B Niemann-Pick disease. J Inherit Metab Dis 2005; 28:13-20.

8 Boven LA, van Meurs M, Boot RG, et al: Gaucher cells demonstrae a distinct macrophage phenotype and resemble alternatively activated macrophages. Am Clin Pathol 2004;122: 359-369.

9 Schroder M, Kaufman RJ: ER stress and the unfolded protein response. Mutat Res 2005; 569:29-63.

10 Gargalovic PS, Gharavi NM, et al: The unfolded protein response is an important regulator of inflammatory genes in endothelial cells. Arterioscler Thromb Vasc Biol 2006;26: 2490-2496.
11 Tessitore A, del P Martin M, Sano R, et al: GM1-ganglioside-mediated activation of the unfolded protein response causes neuronal death in a neurodegenerative gangliosidosis. Mol Cell 2004;15:753-766.

12 Lloyd-Evans E, Pelled D, Riebeling C, et al: Glucosylceramide and glucosylsphingosine modulate calcium mobilization from brain microsomes via different mechanisms. J Biol Chem 2003;278:23594-23599.

13 Korkotian E, Schwarz A, Pelled D, et al: Elevation of intracellular glucosylceramide levels results in an increase in endoplasmic reticulum density and in functional calcium stores in cultured neurons. J Biol Chem 1999;274: 21673-21678.

14 Mikosch P: Gaucher disease and bone. Best Pract Res Clin Rheumatol 2011;25:665-681.

15 Pastores GM: Neuropathic Gaucher disease. Wien Med Wschr 2010;160:605-608.

16 Walton-Bowen K, Mantick N: Gaucher registry annual aggregate data report. 2000.

17 Mehta A: Epidemiology and natural history of Gaucher's disease. Eur J Intern Med 2006; 17:S2-S5.

18 Germain DP: Gaucher's disease: a paradigm for interventional genetics. Clin Genet 2004; 65:77-86.

19 Cox TM: Gaucher disease: clinical profile and therapeutic developments. Biologics 2010;4: 299-313.

20 Grabowski G: Gaucher disease: gene frequencies and genotype/phenotype correlations. Genet Test 1997;1:5-12.

21 Beutler E, Nguyen NJ, Henneberger MW, et al: Gaucher disease: gene frequencies in the Ashkenazi Jewish population. Am J Hum Genet 1993b;52:85-88.

22 Grabowski GA, Horowitz M: Gaucher's disease: molecular, genetic and enzymological aspects. Baillieres Clin Haematol 1997;10: 635-656.
23 Koprivica V, Stone DL, Park JK, et al: Analysis and classification of 304 mutant alleles in patients with type 1 and type 3 Gaucher disease. Am J Hum Genet 2000;66:1777-1786.

24 Kathleen S, Hruska, Mary E, et al: Gaucher disease: mutation and polymorphism spectrum in the glucocerebrosidase gene (GBA). Hum Mutat 2008;29:567-583.

25 Eyal N, Firon N, Wilder S, et al: Three unique base pair changes in a family with Gaucher disease. Hum Genet 1991;87:328-332.

26 Hong CM, Ohashi T, Yu XJ, et al: Sequence of two alleles responsible for Gaucher disease. DNA Cell Biol 1990;9:233-241.

27 Latham T, Grabowski GA, Theophilus BD, et al: Complex alleles of the acid beta-glucosidase gene in Gaucher disease. Am J Hum Genet 1990;47:79-86.

28 Latham TE, Theophilus BD, Grabowski GA, et al: Heterogeneity of mutations in the acid beta-glucosidase gene of Gaucher disease patients. DNA Cell Biol 1991;10:15-21.

29 Tayebi N, Stubblefield BK, Park JK, et al: Reciprocal and nonreciprocal recombination at the glucocerebrosidase gene region: implications for complexity in Gaucher disease. Am J Hum Genet 2003;72:519-534.

30 Zimran A, Sorge J, Gross E, et al: A glucocerebrosidase fusion gene in Gaucher disease: implications for the molecular anatomy, pathogenesis, and diagnosis of this disorder. J Clin Invest 1990b;85:219-222.

31 Sarria AJ, Giraldo P, Perez-Calvo JI, et al: Detection of three rare (G377S, T134P and 1451delAC), and two novel mutations (G195W and $\operatorname{Rec}[1263 \mathrm{del} 55 ; 1342 \mathrm{G}>\mathrm{C}]$ ) in Spanish Gaucher disease patients. Mutation in Brief No. 251. Hum Mutat 1999;14:88.

32 Cormand B, Diaz A, Grinberg D, et al: A new genepseudogene fusion allele due to a recombination in intron 2 of the glucocerebrosidase gene causes Gaucher disease. Blood Cells Mol Dis 2000;26:409-416. 
33 Stone DL, Tayebi N, Orvisky E, et al: Glucocerebrosidase gene mutations in patients with type 2 Gaucher disease. Hum Mutat 2000b; 15: 181-188.

34 Beutler E, Demina A, Gelbart T: Glucocerebrosidase mutations in Gaucher disease. Mol Med 1994;1:82-92.

35 Park JK, Tayebi N, Stubblefield BK, et al: The E326K mutation and Gaucher disease: mutation or polymorphism? Clin Genet 2002;61: 32-34.

36 Walker JM, Lwin A, Tayebi N, et al: Glucocerebrosidase mutation T369M appears to be another polymorphism. Clin Genet 2003;63: 237-238.

37 Torralba MA, Perez-Calvo JI, Pastores GM, et al: Erratum: identification and characterization of a novel mutation c.1090G4T (G325W) and nine common mutant alleles leading to Gaucher disease in Spanish patients. Blood Cells Mol Dis 2001a;27:713.

38 Torralba MA, Perez-Calvo JI, Pastores GM, et al: Identification and characterization of a novel mutation c.1090G4T (G325W) and nine common mutant alleles leading to Gaucher disease in Spanish patients. Blood Cells Mol Dis 2001b;27:489-495.

39 Hruska K, LaMarca ME, Sidransky E: Gaucher disease: molecular biology and genotype-phenotype correlations; in Futerman AH, Zimran A (eds): Gaucher Disease. Boca Raton, CRC Press, 2007; pp 13-48.

40 Sorge J, Gelbart T, West C, et al: Heterogeneity in type I Gaucher disease demonstrated by restriction mapping of the gene. Proc Natl Acad Sci USA 1985a;82:5442-5445.

41 Zimran A, Gelbart T, Beutler E: Linkage of the PvuII polymorphism with the common Jewish mutation for Gaucher disease. Am J Hum Genet 1990a;46:902-905.

42 Beutler E, West C, Gelbart T: Polymorphisms in the human glucocerebrosidase gene. Genomics 1992b;12:795-800.

43 Amaral O, Marcao A, Pinto E, et al: Distinct haplotype in non-Ashkenazi Gaucher patients with N370S mutation. Blood Cells Mol Dis 1997;23:415-416.

44 Cormand B, Grinberg D, Gort L, et al: Molecular analysis and clinical findings in the Spanish Gaucher disease population: putative haplotype of the N370S ancestral chromosome. Hum Mutat 1998a;11:295-305.

45 Demina A, Beutler E: Six new Gaucher disease mutations. Acta Haematol 1998;99:80-82.

46 Lacerda L, Amaral O, Pinto R, et al: The N370S mutation in the glucocerebrosidase gene of Portuguese type 1 Gaucher patients: linkage to the PvuII polymorphism. J Inherit Metab Dis 1994;17:85-88.

47 Rockah R, Narinsky R, Frydman M, et al: Linkage disequilibrium of common Gaucher disease mutations with a polymorphic site in the pyruvate kinase (PKLR) gene. Am J Med Genet 1998;78:233-236.
48 Rodriguez-Mari A, Diaz-Font A, Chabas A, et al: New insights into the origin of the Gaucher disease causing mutation N370S: extended haplotype analysis using the 5GC3.2, 5470 G/A, and ITG6.2 polymorphisms. Blood Cells Mol Dis 2001;27:950-959.

49 Lau EK, Tayebi N, Ingraham LJ, et al: Two novel polymorphic sequences in the glucocerebrosidase gene region enhance mutational screening and founder effect studies of patients with Gaucher disease. Hum Genet 1999;104:293-300.

50 Sidransky E, Nalls MA, Aasly JO, et al: Multicenter analysis of glucocerebrosidase mutations in Parkinson's disease. N Engl J Med 2009;361:1651-1661.

51 de Rijk MC, Launer LJ, Berger K, et al: Prevalence of Parkinson's disease in Europe: a collaborative study of population-based cohorts. Neurologic Diseases in the Elderly Research Group. Neurology 2000;54(11 suppl 5):S21S23.

52 Bekris LM, Mata IF, Zabetian CP: The genetics of Parkinson disease. J Geriatr Psychiatry Neurol 2010;23:228-242.

53 Zimran A, Neudorfer O, Elstein D: The glucocerebrosidase gene and Parkinson's disease in Ashkenazi Jews. N Engl J Med 2005;352: 728-731.

54 Aharon-Peretz J, Rosenbaum H, GershoniBaruch R: Mutations in the glucocerebrosidase gene and Parkinson's disease in Ashkenazi Jews. N Engl J Med 2004;351:1972-1977.

55 Goker-Alpan O, Schiffmann R, LaMarca ME, et al: Parkinsonism among Gaucher disease carriers. J Med Genet 2004;41:937-940.

56 Halperin A, Elstein D, Zimran A: Increased incidence of Parkinson disease among relatives of patients with Gaucher disease. Blood Cell Mol Dis 2006;6:426-428.

57 Mitsui J, Mizuta I, Toyoda A, et al: Mutations for Gaucher disease confer high susceptibility to Parkinson disease. Arch Neurol 2009;66: 571-576.

58 Lesage S, Anheim M, Condroyer C, et al: Large-scale screening of the Gaucher's disease-related glucocerebrosidase gene in Europeans with Parkinson's disease. Hum Mol Genet 2011;20:202-210.

59 Kamath RS, Lukina E, Watman N, et al: Skeletal improvement in patients with Gaucher disease type 1: a phase 2 trial of oral eliglustat. Skeletal Radiol 2014;43:1353-1360.

60 Rosenthal DI, Scott JA, Barranger J, et al: Evaluation of Gaucher disease using magnetic resonance imaging. J Bone Joint Surg Am 1986;68:802-808

61 Mistry PK, Weinreb NJ, Kaplan P, et al: Osteopenia in Gaucher disease develops early in life: response to imiglucerase enzyme therapy in children, adolescents and adults. Blood Cells Mol Dis 2011;46:66-72.

62 Maas M, Poll LW, Terk MR: Imaging and quantifying skeletal involvement in Gaucher disease. Br J Radiol 2002;75(suppl 1):A13A24.
63 Kuter DJ, Mehta A, Hollak CEM, et al: Miglustat therapy in type 1 Gaucher disease: clinical and safety outcomes in a multicenter retrospective cohort study. Blood Cells Mol Dis 2013;51:116-124.

64 Elstein D, Hollak C, Aerts JM, et al: Sustained therapeutic effects of oral miglustat (Zavesca, N-butyldeoxynojirimycin, OGT 918) in type I Gaucher disease. J Inherit Metab Dis 2004; 27:757-766.

65 Heitner R, Elstein D, Aerts J, et al: Low-dose $\mathrm{N}$-butyldeoxynojirimycin (OGT 918) for type I Gaucher disease. Blood Cells Mol Dis 2002; 28:127-133.

66 Pastores GM, Giraldo P, Cherin P, et al: Goaloriented therapy with miglustat in Gaucher disease. Curr Med Res Opin 2009;25:23-37.

67 Elstein D, Dweck A, Attias D, et al: Oral maintenance clinical trial with miglustat for type I Gaucher disease: switch fromor combination with intravenous enzyme replacement. Blood 2007;110:2296-2301.

68 Pastores GM, Elstein D, Hrebicek M, et al: Effect of miglustat on bone disease in adults with type 1 Gaucher disease: a pooled analysis of three multinational, open-label studies. Clin Ther 2007;29:1645-1654.

69 Martinez-Arias R, Calafell F, Mateu E, et al: Sequence variability of a human pseudogene. Genome Res 2001a;11:1071-1085.

70 Zimran A: How I treat Gaucher disease. Blood 2011;118:6.

71 Barton NW, Brady RO, Dambrosia JM, et al: Replacement therapy for inherited enzyme deficiency - macrophage-targeted glucocerebrosidase for Gaucher's disease. N Engl J Med 1991;324:1464-1470.

72 Lukina E, Watman N, Arreguin EA, et al: Improvement in hematological, visceral, and skeletal manifestations of Gaucher disease type 1 with oral eliglustat tartrate (Genz112638 ) treatment: 2 -year results of a phase 2 study. Blood 2010;116:4095-4098.

73 Deegan PB, Cox TM: Imiglucerase in the treatment of Gaucher disease: a history and perspective. Drug Des Devel Ther 2012;6:81106.

74 Horowitz M, Wilder S, Horowitz Z, et al: The human glucocerebrosidase gene and pseudogene: structure and evolution. Genomics 1989;4:87-96.

75 Ron I, Horowitz M: ER retention and degradation as the molecular basis underlying Gaucher disease heterogeneity. Hum Mol Genet 2005; 14:2387-2398.

76 Kornhaber GJ, Tropak MB, Maegawa GH, et al: Isofagomine induced stabilization of glucocerebrosidase. Chem Biochem 2008;9: 2643-2649.

77 Maegawa GH, Tropak MB, Buttner JD, et al: Identification and characterization of ambroxol as an enzyme enhancement agent for Gaucher disease. J Biol Chem 2009;284: 23502-23516. 
78 Laoag-Fernandez JB, Fernandez AM, Maruo T: Antenatal use of ambroxol for the prevention of infant respiratory distress syndrome. J Obstet Gynaecol Res 2000;26:307-312.

79 Peters C, Krivit W: Hematopoietic stem cell therapy, stem cells and gene therapy; in Futerman AH, Zimran A (eds): Gaucher Disease. Boca Raton, CRC Press, 2007, pp 423-438.

80 Enquist IB, Nilsson E, Månsson JE, et al: Successful low-risk hematopoietic cell therapy in a mouse model of type 1 Gaucher disease. Stem Cells 2009;27:744-752.

81 Grabowski GA: Gaucher disease and other storage disorders. Hematol Am Soc Hematol Educ Program 2012;1:13-18.

82 Brautbar A, Abrahamov A, Hadas-Halpern I, et al: Gaucher disease in Arab patients at an Israeli referral clinic. Isr Med Assoc J 2008; 10 : 600-602.
83 Chaabouni M, Aoulou H, Tebib N, et al: Gaucher's disease in Tunisia (multicenter study). Rev Med Interne 2004;25:104-110.

84 Cherif W, Ben Turkia H, Ben Rhouma F, et al: Gaucher disease in Tunisia: high frequency of the most common mutations. Blood Cells Mol Dis 2009;43:161-162.

85 Alfonso P, Cenarro A, Pérez-Calvo JI, et al Mutation prevalence among 51 unrelated Spanish patients with Gaucher disease: identification of 11 novel mutations. Blood Cells Mol Dis 2001;27:882-891.

86 Filocamo M, Mazzotti R, Stroppiano M, et al: Analysis of the glucocerebrosidase gene and mutation profile in 144 Italian Gaucher patients. Hum Mutat 2002;20:234-235.
87 Rodriguez-Mari A, Diaz-Font A, Chabás A, et al: New insights into the origin of the Gaucher disease-causing mutation N370S: extended haplotype analysis using the 5GC3.2, $5470 \mathrm{G} / \mathrm{A}$, and ITG6.2 polymorphisms. Blood Cells Mol Dis 2001;27:950-959.

88 Ben Turkia H, Riahi I, Azzouz H, Ladab S, et al: Phenotype and mutational spectrum in Tunisian pediatric Gaucher disease. Tunis Med 2010;88:158-162.

89 Ben Rhouma F, Kallel F, Kefi R, et al: Adult Gaucher disease in southern Tunisia: report of three cases. Diagn Pathol 2012;7:4.

90 Dandana A, Ferchichi S, Khedhiri S, et al: Biochemical and molecular diagnosis of Gaucher disease in Tunisia. Ann Biol Clin (Paris) 2007; 65:647-652. 